\title{
Semantic priming without association: A meta-analytic review
}

\author{
MARGERY LUCAS \\ Wellesley College, Wellesley, Massachusetts
}

\begin{abstract}
A meta-analysis of 26 studies indicated that automatic semantic priming can occur without association. Priming did not vary substantially with differences in variables that affect automatic versus strategic processing, such as time spent processing the prime and target, relationship proportion, and task (except that average effects were smaller in the naming task). Although category coordinates were investigated in the majority of studies, synonyms, antonyms, and script relations also demonstrated priming; functional relations showed greater priming, and essential and perceptual relations showed less. The average effect size for semantic priming was smaller than that for associative priming, suggesting that there is an "associative boost" from adding an associative relationship to a semantic one. The implications of these findings for the modularity thesis and for models of semantic priming are discussed.
\end{abstract}

The phenomenon of semantic priming is a rich source of information about the mental lexicon. In semantic priming, a target word (such as butter) is responded to more quickly after presentation of a related prime word (such as bread) than after presentation of an unrelated prime. The semantic priming effect has been used to investigate how words are accessed, comprehended, and integrated with other words in sentence understanding. Although most researchers agree that semantic priming is a valuable tool for studying these processes, they have not always agreed about the types of relationships that are primed. In the earliest studies of semantic priming (Meyer \& Schvaneveldt, 1971; Meyer, Schvaneveldt, \& Ruddy, 1975), semantic priming was actually associative priming: prime-target pairs were drawn from norms of word association. Fischler (1977) was the first to unconfound semantic and associative relationships, by looking at priming for both associative pairs and pairs that were semantically related but not associated. Fischler found that semantically related pairs that were not also associated did exhibit priming. Attempts to replicate this finding, however, have led to mixed results, with some studies resulting in priming for semantic pairs without association and others not. Differences in methodologies and stimulus choice have contributed to the controversy over

I am very grateful to all the authors who generously supplied information that was not available in their published work: Christine Chiarello, Helen Moss, Kate Nation, Diane Pecher, Manolo Perea, Eva Rosa, Jennifer Shelton, Li Hai Tan, John Williams, and Piene Zwitserlood. This meta-analysis would have been far less interesting and comprehensive without their kind cooperation. I also thank Andrea Levitt, Ken Paap, and two anonymous reviewers for their many helpful comments and suggestions on an earlier draft of this manuscript. Correspondence concerning this article should be addressed to the author at the Department of Psychology, Wellesley College, Wellesley, MA 02481 (e-mail:mlucas@wellesley.edu). the existence of purely semantic (as opposed to associative) priming.

At stake is our understanding of the organization of the mental lexicon. According to one of the most popular theories of lexical processing, the spreading activation theory of Collins and Loftus (1975), information about words and their meanings is stored in separate networks. One network is purely lexical; that is, it contains only phonemic and orthographic information about words. The other network is purely semantic and contains all concepts, including those linked to the word forms in the lexical network. Nodes in the lexical network are connected to each other on the basis of phonological and orthographic similarity, whereas nodes in the semantic network are connected to each other on the basis of semantic similarity. According to this theory, links between the lexical and the semantic networks are as easily activated as are those within a network.

This theory, in which lexical and conceptual knowledge are interactive, can be contrasted with a modular theory as proposed by Fodor (1983), Forster (1979), and others. On the modular view, priming within the lexical network of phonological and orthographic information is based on associative links which connect words that are often contiguous (e.g., needle and thread) but that may share few if any semantic features. This intralexical priming is automatic and unaffected by feedback from the conceptual network. Connections within the conceptual network (e.g., category coordinates that share features, such as ROBIN and EAGLE) may prime each other, but this priming cannot provide feedback to the lexical network to facilitate the activation of related word forms (such as robin). Facilitation arising from within the conceptual network can only influence later stages in processing such as the stage that selects the most likely lexical candidate from among a number of activated representations or the stage that attempts to integrate a 
lexical representation with others that have preceded it. In contrast to the automatic activation of associative links in the lexical network, these postlexical priming processes are the result of strategies adopted by the subject. One motivation for looking for purely semantic priming is to test this intralexical priming hypothesis according to which automatic priming can only be associative, not semantic or conceptual.

The outcome of this controversy over the organization of lexical and conceptual knowledge will profoundly influence the way in which we think about word meaning and lexical processing. If some version of the Collins and Loftus (1975) story is true, then intuitions about the lexicon are satisfied: The meaning of a word is integrated with its word form in such a way that the meanings or concepts associated with words are activated along with phonological, orthographic, and co-occurrence information. Seeing the word robin causes the concept of ROBIN to be activated along with related concepts like EAGLE and SPARROW. These conceptual activations can then feed back to activate the word forms connected to those concepts and these word forms will then be easily recognized, named, and so forth. But if the integration of word form and word meaning is limited in the way suggested by the intralexical priming hypothesis, then, counterintuitively, meaning is not part of the lexicon per se. Meaning is connected to the lexicon via links from the lexical to the conceptual network, but interactions between the two networks are limited.

On this view, automatic priming cannot tell us about the organization of semantic information, only about cooccurrence contingencies among words. This surprising result could occur because priming has multiple sources; it can reflect automatic processes as well as strategic (postlexical) processes. Because strategic processes can find relationships on the fly, depending on the demands of particular tasks, it is generally thought that only automatic processes reliably reflect the long-lasting organizational structure of semantic networks (Seidenberg, Waters, Sanders, \& Langer, 1984). Therefore, teasing out the effects due to automatic versus strategic processes will be part of the work of this review.

Recently, there has been some controversy over whether or not any priming, including associative priming, is automatic (see Boronat, 1998, for a review). Automaticity is usually defined by a set of criteria that include fast processing of a stimulus without awareness, attention, or intention (Posner \& Snyder, 1975). A number of studies, however, have shown that semantic priming can only occur if subjects are attending to the prime (Boronat, 1998; Henik, Friedrich, \& Kellogg, 1983; Paap \& Newsome, 1981; Smith, 1979; Stolz \& Besner, 1999). These findings have led some to conclude that semantic priming cannot be automatic (Stolz \& Besner, 1999), although others argue that the findings indicate that automatic priming requires attention (Logan, 1988). Because all but one of the studies in this review (Kacinik, 1998) required the subject to attend to the prime, the data in this review cannot be used to address this particular controversy. Moreover, whether or not automaticity requires attention is tangential to the main purpose of this review, which is to determine whether or not semantic priming can occur without association. So, the present investigation of automatic semantic priming will focus on evidence from tests of less controversial aspects of automaticity, such as speed of processing and opportunity to use strategies.

Differences in variables affecting automatic and strategic processing, such as the type of task used to measure priming, the difference in prime and target onset time, and the proportion of related and unrelated targets in stimulus lists, may have contributed to the mixed results in the literature on semantic priming. All of these possibilities will be evaluated in this review. Another difficulty may be the low power of studies using small sample sizes to detect a semantic priming effect. With meta-analysis, which has been shown to be a powerful technique for making meaningful, quantitative comparisons across experiments, it is possible to increase the power to detect an effect by combining effect sizes from many studies (Glass, McGaw, \& Smith, 1981; Light \& Pillemer, 1984). In this review, meta-analysis will be used to assess the following three hypotheses: (1) that semantic priming without association exists, (2) that semantic priming is automatic, and (3) that there is a "boost" in priming from adding an associative relationship to an existing semantic one. Differences in priming among different types of semantic relationships and the nature of the difference in semantic and associative relationships will also be investigated. The implications of the results for the modularity thesis and for models of semantic priming will be discussed.

\section{METHOD}

\section{Selection of Studies}

The most important criterion for a study's inclusion in the meta-analysis was that its authors must have used purely semantically related prime-target pairs as stimuli, controlling for association value so that semantically related pairs had no or very little associative relationship. Methods for controlling for association varied considerably across papers. Some authors simply checked one or more published association norms: If the primetarget pairs were not listed, the pairs were deemed nonassociative. Other authors used their own word association test to eliminate associated targets; experimenters using this technique also varied in their criteria for inclusion of a prime-target pair as unrelated. Some eliminated a target only if it appeared as one of the first associates; others eliminated a target that appeared anywhere as an associate. Some authors did not specify how association was ruled out, merely stating that it was. Some authors claimed that their prime-target pairs had no association value; others tolerated weak association values. Such a variety of techniques and criteria occurred across all the studies under consideration that it would not have been fruitful to compare studies on this factor. So, for the purpose of 
inclusion in the meta-analysis, the only relevant selection criterion was whether or not the author(s) explicitly stated that some attempt (of any kind) was made to control for associative relationships among prime-target pairs. The implications of these types of differences among stimulus choices for the study of semantic priming will be considered later in the Conclusions section.

Other criteria for inclusion in the review included the following: (1) Only priming studies with reaction time as a dependent measure were included. Studies with memory measures were not included because the interest was in immediate access of lexical information. Other types of immediate decision tasks (such as deciding whether a target word belongs to a certain category) were excluded because they required the subject to focus explicitly on the meaning of the stimuli, which would strongly encourage strategic processing. Priming tasks included lexical decision, naming, or color naming (also known as the Stroop task). In a lexical decision task, subjects hear or read a string of letters and indicate by a button press whether or not the string is a word. Subjects are faster to make a lexical decision if the string is preceded by a related word than if it is not. For example, they are faster to say that butter is a word if they have just seen bread than if they have just seen an unrelated word such as board. In a naming task, subjects are asked to say the target word out loud. Latency to initiate this naming response is the measure of priming. In a color-naming task, targets are printed in different colors and subjects must name the color of the target, ignoring the word itself. Interference from the target word to naming the color is the measure of priming. (2) Studies with primetarget pairs embedded in sentence contexts were excluded because of concerns that cumulative context effects might cause different effects from those found in isolated pairs (but see Lucas, 1999, for a review of similar issues involved in sentence processing). The exception to this was Zwitserlood and Schriefers (1995), who embedded their primes in what they called "carrier phrases" such as "You are now going to listen to ..." and "Now you will hear ..." This study was included because the carrier phrases contained no semantic or syntactic information that might aid in the recognition of the prime or target word and because the study otherwise met the criteria for inclusion. (3) Only studies of normal adult populations were included, although the results of the normal adult control populations employed in studies of brain-damaged patients or children were sometimes included. Although studies of the latter populations can be the source of much useful information about the organization of the lexicon, they were excluded because response times in impaired and unimpaired lexical processing are not readily comparable (likewise for reaction times in mature and immature populations). Because the issues raised by the study of impaired lexical processing are more complex and problematic than the issues raised by the study of unimpaired lexical processing alone, the former really needs its own analysis.

\section{Procedure}

The First Search database was canvassed for references by using semantic priming as the keyword. The literature review and discussion sections of published papers were also searched for relevant references, and work was solicited from authors working in this area. With these search procedures, 36 references that met the criteria above were identified: 29 journal articles, 4 dissertations, 1 master's thesis, and 2 unpublished manuscripts. Not all of the studies included information about variability from which to calculate an effect size. Whenever possible, this information was obtained by contacting the author. In the end, a total of 26 studies out of the original 36 could be used in the meta-analysis; these met the criteria stated in the previous section and also provided information from which to calculate an effect size.

Many of these references included multiple experiments that involved the manipulation of a number of variables thought to affect semantic processing, such as task or timing of target presentation. Moreover, in individual experiments repeated measures were often used to obtain data for more than one variable. Because these within-study and within-experiment manipulations concerned variables of critical importance to the hypotheses that motivated the review, each manipulation within an experiment was included in the meta-analysis and counted as a single "observation." There were a total of $116 \mathrm{ob}-$ servations across the 26 studies. However, repeated measures mean observations are not independent, and this is problematic when one is testing for heterogeneity of variance (Rosenthal \& Rubin, 1982). So the results are reported both by study and by observation.

\section{RESULTS}

\section{Effect Sizes}

Effect sizes were calculated for the amount of priming for targets that appeared after purely semantically related primes and for targets that appeared after associatively related primes, when the latter were included as stimuli. Effect sizes were calculated as follows. Each observation was converted to a standard effect size $(d)$. Cohen's $d$ was calculated using Cohen's (1977) formula for the estimate of the pooled standard deviation. When means and standard deviations were not provided, the square root of the $M S_{\mathrm{e}}$ of the main effect of relatedness (the comparison of related targets vs. control targets) was used to estimate the pooled standard deviation (Glass et al., 1981). Only the results of subject analyses were used in this meta-analysis, for two reasons. First, results of subject analyses were the only variability data available across all 26 studies. Standard deviations are typically not reported for item analyses, and the reporting of $M S_{\mathrm{e}} \mathrm{s}$ is a relatively recent phenomenon, inconsistently practiced. Although authors sometimes could provide information about variability that was not reported in their published papers, this information was not available when studies were more than 5 years old. Second, sub- 
ject analyses should be adequate for meta-analysis. Although it is true that the results of any single experiment may be skewed by a handful of items (hence the rationale for an item analysis), a meta-analysis averages effects over a number of experiments. The result is a more reliable $d$ estimate than can be obtained from any one of them.

In addition to effect sizes for individual observations, an overall average $d$ was calculated for each study by calculating the mean of all individual effect sizes in a study. A positive $d$ indicates a priming effect, with one exception. In the Stroop or color-naming task, inhibition rather than facilitation of the related targets indicates priming. In this case, priming would be indicated by a negative $d$ in the one study that used the color-naming task. For ease of comparison with other studies, the sign of the $d$ for color-naming results was reversed so that a positive $d$ in that study could be interpreted as facilitation rather than inhibition. Effect sizes for each study included in the review are listed in Table 1.

\section{Central Tendency and Variability}

For the 116 observations, the average $d$ for semantic priming was .26, which is a little more than half the effect size found for associative priming (73 observations), which was .51 . The average $d$ by study was .30 for semantic priming ( 26 studies) and .49 for associative priming (20 studies). The total number of subjects across all 116 observations was 3,882 . Because observations sometimes involved repeated measures, some of these subjects accounted for more than one observation. Corrected for repeated measures, the total number of subjects across all the 26 studies was 2,243 . Because the range of subjects was so varied (from 7 to 359 subjects in a single study), the weighted mean for each observation was also calculated, using the formula in Hedges and Olkin (1985). The weighted average $d$ for semantic priming, by observation, was .25 , and for associative priming, it was .49 . By study, the weighted average $d s$ were .29 for semantic priming and .47 for associative priming. In subsequent analyses, only the weighted mean effect sizes will be reported.

Fifty percent of the observations for semantic priming that were in the midrange had effect sizes between .05 and .36 ( 25 th and 75 th percentile values); this range was .17 to .59 for associative priming. For the studies, the range was somewhat narrower-between .09 and .36 for semantic priming and between .18 and .54 for associative priming.

These results indicate that the effect of semantic priming, while small, is nonetheless clearly present in the studies reviewed. The small effect size for semantic priming suggests one reason for the failures of some studies to yield statistically significant semantic priming effects. A power analysis indicates that one would need more than twice as many subjects $(N=188)$ to find statistically significant semantic priming as one would need to find statistically significant associative priming $(N=$ 72) with power of .80 and an alpha level of .05.

\section{Heterogeneity of Variance}

Although effect sizes were consistently positive for semantic priming, there is quite a bit of variability across studies. A critical question concerns whether this variability represents random sampling variation around a

Table 1

Weighted Effect Sizes of Semantic and Associative Priming Effects (by Study)

\begin{tabular}{lcc} 
& Semantic Priming & Associative Priming \\
\hline Fischler (1977) & $d$ & $d$ \\
Kruesi (1979) & .79 & 1.17 \\
Seidenberg, Waters, Sanders, \& Langer (1984) & .34 & .5 \\
Chertkow, Bub, \& Seidenberg (1989) & .96 & .87 \\
Scholten (1991) & .08 & .14 \\
Chiarello \& Richards (1992) & .29 & .35 \\
Shelton \& Martin (1992) & .09 & - \\
Ostrin \& Tyler (1993) & .19 & .52 \\
Shelton (1993) & .96 & 1.16 \\
Williams (1994) & -.03 & .18 \\
Moss, Ostrin, Tyler, \& Marslen-Wilson (1995) & .41 & -.05 \\
Zwitserlood \& Schriefers (1995) & .50 & 1.05 \\
Abernethy \& Coney (1996) & .25 & - \\
Williams (1996) & .25 & - \\
Djojomihardjo (1997) & .21 & .37 \\
Moss, McCormick, \& Tyler (1997) & -.01 & .82 \\
Perea \& Gotor (1997) & .46 & .26 \\
Perea, Gotor, \& Nacher (1997) & .18 & .15 \\
Kacinik (1998) & .09 & .27 \\
McRae \& Boisvert (1998) & .25 & .18 \\
Pecher, Zeelenberg, \& Raaijmakers (1998) & .34 & - \\
Perfetti \& Tan (1998) & .07 & .52 \\
Thompson-Schill, Kurtz, \& Gabrieli (1998) & .28 & - \\
Nation \& Snowling (1999) & .17 & .22 \\
Lucas (in press) & .36 & .54 \\
Perea \& Rosa (1998) & .05 & - \\
\hline
\end{tabular}


single, normally distributed $d$ value of roughly .3 or reflects the existence of two (or more) underlying populations that differ on some background factor or factors (Light \& Pillemer, 1984). These possibilities were investigated in a test for heterogeneity of variance. In such tests, effect sizes should be independent (Rosenthal \& Rubin, 1982), so only the effect sizes from the studies were used. The test statistic provided by Hedges and Olkin (1985) was used. This test statistic is approximately distributed as a chi square with $K-1 d f$, where $K$ is the number of effect sizes. When Hedges and Olkin's formula was applied to the study effect sizes for semantic priming, it yielded an approximate chi square of 43.09 with $25 d f$, significant at $p<.02$. Therefore, a search for moderator variables seemed warranted. The variables chosen were those relevant to the hypotheses described in the introduction.

\section{Is Semantic Priming Automatic?}

The results of the meta-analysis indicate that there can be semantic priming without association, but it is possible that this semantic priming, when it was observed, was due to strategic rather than automatic processing. Strategic processes take time to develop and are thought to be found only during later stages of processing - that is, with SOAs greater than $250 \mathrm{msec}$ (Posner \& Snyder, 1975) or when reaction times are slow (Williams, 1996). A longer amount of time to process the prime and target might lead to greater semantic priming because of the additional time available for strategic processes such as integrating the prime with the target (DeGroot, 1984; Neely, 1977; Seidenberg et al., 1984). A similar argument can be made for the influence of relatedness proportion. When the proportion of related targets to unrelated targets is relatively high, there is a greater likelihood that subjects will notice the relationship between prime and target, which will in turn facilitate the use of strategic processes (DeGroot, 1984; Keefe \& Neely, 1990; Neely, 1977; Seidenberg et al., 1984). Finally, some tasks are considered more likely to show strategic effects than others. The lexical decision task is thought to be particularly susceptible to the effects of strategic processing (Balota $\&$ Chumbley, 1984; Seidenberg et al., 1984) unless the primes are masked or the primes and targets are presented continuously instead of paired (Balota, 1983; McNamara \& Altarriba, 1988; Shelton \& Martin, 1992; Williams, 1994). Naming is less susceptible to strategic processing but can show effects of expectancy (Huttenlocher \& Kubicek, 1983; Keefe \& Neely, 1990). It follows that if semantic priming without association is due solely to strategic processes, the effects should be larger under conditions that enable strategic processing - that is, when SOAs are long, reaction times are slow, and relatedness proportions are high. Effects should be smaller under conditions that obstruct strategic processing-that is, when the masked or continuous lexical decision task is used.

\section{Effects of Task}

Semantic priming was evaluated in four different tasks: naming, paired lexical decision (LD), lexical decision with a mask (MLD), and serial or continuous lexical decision (CLD). The Stroop task was not included, because it had been used in only one study (Shelton, 1993), and that made it impossible to draw any generalizations about Stroop and semantic priming.

If semantic priming is due to strategic processes, there should be little if any priming effect in MLD or CLD, tasks that obstruct strategic processing. However, for the majority of the studies, and contrary to expectations, the effects were surprisingly similar across tasks. Some tasks that are purported to be purer measures of automatic priming showed similar effects to that of paired lexical decision. Out of 45 observations obtained using lexical decision, the average $d$ was .33 , which was close to the average $d$ for CLD $(d=.27 ; 16$ observations $)$ and for $\operatorname{MLD}(d=.26 ; 23$ observations). There was a greater spread across tasks in the study comparisons; nonetheless there was still a small effect of semantic priming in the latter two tasks. The average $d$ for paired LD was .39 (16 studies); for MLD, .28 (5 studies); and for CLD, .18 (6 studies). Only naming showed evidence of a smaller semantic priming effect both by observations $(d=.16$; 29 observations) and by studies ( $d=.18 ; 9$ studies).

The smaller effects for naming are not surprising, because naming effects are usually smaller than lexical decision effects (Hodgson, 1991). Some possible reasons for this will be discussed later, in the section on response speed. For the present purposes, it is noteworthy that the paired LD task did not produce substantially larger effects than did MLD or CLD. What the pattern seems to show is that paired LD modestly inflates an existing small semantic priming effect. One caveat is that the number of studies with MLD and CLD was appreciably smaller than those with paired LD. More studies done with these tasks are called for; nonetheless there is no evidence in these effect sizes that semantic priming is eliminated or substantially reduced when CLD or MLD is used.

\section{Relatedness Proportion}

Relatedness proportion can be calculated in one of two ways: as the proportion of related words for all word-prime/word-target trials or as the proportion of related words for all trials including those with nonword targets. Following Neely (1991), the former measure is used here. A relatedness proportion of .33 or less was categorized as low; one of greater than .33 was categorized as high (also from Neely, 1991). If semantic priming is due to strategic processing, there should be little or no semantic priming with low relatedness proportions. However, relatedness proportion did not influence the size of effect in those studies for which the measure could be calculated. The average $d$ for low relatedness proportions was .26 (48 observations), which was almost 
identical to the average $d$ of .25 (60 observations) for high relatedness proportions. The corresponding numbers for studies were .22 ( 12 studies) for low relatedness proportions versus 31 (15 studies) for high relatedness proportions, indicating only a slight advantage for the larger relatedness proportions. In any case, there was still a semantic priming effect even in the low relatedness proportion conditions of experiments.

\section{Stimulus Onset Asynchrony}

Assessing the effects of SOA was problematic because SOA was not always reported; sometimes interstimulus interval (ISI) was reported instead. This was most likely to be true of the studies in which auditory primes or CLD were used. This problem could be overcome with the CLD task, because in that task all stimuli are targets and all targets remain in view until subjects respond. Therefore, when CLD was the task, SOA could be estimated by using the average reaction time to control targets as an estimate of prime duration and then adding the ISI to it. No comparable estimate of prime duration was available for auditory primes unless authors reported average prime duration, because no response to auditory primes was required. So unless authors reported SOAs, the data from experiments done with auditory primes were excluded.

Facilitation of related targets without inhibition of unrelated targets is thought to indicate automatic processing; this pattern of responses has been found with SOAs of 250 msec or less in many studies (Neely, 1991). Accordingly, observations and studies were partitioned into two categories. One category comprised SOAs of $250 \mathrm{msec}$ or less (a category in which primarily automatic processes are found), and the other, SOAs above $250 \mathrm{msec}$ (a category in which primarily controlled processes are found). There was, however, no appreciable difference in effect sizes between these two categories. For SOAs shorter than $250 \mathrm{msec}, d=.23$ (49 observations) and $d=.25$ (12 studies). For SOAs longer than $250 \mathrm{msec}, d=.17$ (52 observations) and $d=.24$ (16 studies). Therefore, there was still semantic priming with SOAs short enough for automatic processing.

\section{Response Speed}

Observations and studies were partitioned into fast ( $500 \mathrm{msec}$ or less) and slow (greater than $500 \mathrm{msec}$ ) average reaction times (the cutoffs were based on suggestions by Williams, 1996). Fast response times should be associated with automatic priming; slow response times, with strategic priming. Because in the Stroop task slower rather than faster reaction times signal automatic priming, the Stroop study of Shelton (1993) was not included in the following analysis.

Response time had a dramatic effect on effect sizes. When reaction times were faster than $500 \mathrm{msec}$, semantic priming vanished. The average $d$ was only .03 over 14 observations and .05 over 5 studies. In contrast, priming was clearly available with slower response speeds; average $d=.30$ by both observations (99) and studies (11).
This analysis gave the first indication that semantic priming might not be automatic. However, it seemed odd that the other three indicators of automaticity (SOA, task, and relatedness proportion) showed evidence for automatic semantic priming. If one looks more closely at the relatively few observations in the fast response speed category, 10 out of the 14 observations were from the naming task. Hodgson (1991) has remarked on the consistently low priming effects (in the range of only a few milliseconds of facilitation) in naming studies when conditions make it difficult to use attentional strategies. Hodgson argues that there is no automatic semantic activation in the lexicon and that the extremely small priming effects in naming are the result of residual attentional processes. Another possibility, however, is that naming is not as sensitive as other tasks to activation in the lexicon. This conclusion does not seem unreasonable in light of the finding that semantic priming occurs with both CLD and MLD, tasks that are also supposed to discourage strategic processing. So, it may be that the lack of an effect in the fast response speed category was due more to the prevalence of naming data in that category than to a dearth of semantic priming.

It is also possible that response time is an insufficient measure of the total time available to the subject to develop strategies. If the SOA was very long, there might be ample opportunity to anticipate possible targets so that the prime and target could be rapidly integrated. If so, reaction times might be fast even though strategies were successfully deployed. Conversely, if SOAs were very brief (and some SOAs were as brief as $50 \mathrm{msec}$ ), even a slowed response might not provide adequate time to integrate a prime and its target; if so, slow responses following very brief SOAs might not always reflect the use of strategies. For these reasons, the combination of SOA and response speed would be a better measure of how much total processing time was available for developing strategies. Accordingly, two categories of total processing time were created. The category of short total processing times included effects from experiments with combined SOAs and average response times of $750 \mathrm{msec}$ or less. The figure of $750 \mathrm{msec}$ was chosen as the cutoff by adding the cutoff for fast response times $(500 \mathrm{msec})$ to the cutoff for short SOAs $(250 \mathrm{msec})$. It is unlikely that strategic processing could occur with any combination of SOA and response time less than $750 \mathrm{msec}$. The category of long total processing times included effects from experiments with combined SOAs and response times that were greater than $750 \mathrm{msec}$. There was no difference in long versus short total processing times: For total processing times less than or equal to $750 \mathrm{msec}$, the average $d$ was .22 (21 observations) and .21 (5 studies). For total processing times greater than $750 \mathrm{msec}$, effect sizes were very similar: average $d=.20$ (77 observations) and .23 ( 20 studies).

In summary, by three measures of automaticity (task, relatedness proportion, and time to process the prime and target), the evidence supports automatic priming for 
semantically related word pairs that are not also associated. The meta-analysis has shown that semantic priming is automatic because the magnitude of the semantic priming effect is not affected by variables that allow strategic processing (SOA, task, etc.). This is somewhat curious, because strategic processes have long been known to influence associative priming. For example, there is both inhibition and a relatedness proportion effect for associative pairs at SOAs greater than $250 \mathrm{msec}$ (Neely, 1991). Are there, then, two kinds of priming, purely semantic priming, which is automatic, and purely associative priming, which is strategic, as suggested by Thompson-Schill, Kurtz, and Gabrieli (1998)? I think it would be premature to draw this conclusion. The metaanalysis indicates that semantic priming can be automatic, because priming occurred with short SOAs and with tasks like CLD that make strategic processing unlikely. But the evidence is weaker that semantic priming cannot also be strategic under the right circumstances. One area of uncertainty concerns the finding of no difference between effect sizes in the categories of low versus high relatedness proportions. Relatedness proportions of greater than .33 were categorized as high proportions, but most studies had relatedness proportions on the low end of the range. Only one experiment had a relatedness proportion higher than .5 , which would provide the strongest test of the possible effects of that variable. It may be that evidence for proportionality effects will turn up with more extreme relatedness proportions than were found in this set of studies, especially at longer SOAs. Also, evidence for inhibition (another strong indicator of strategic processing) could not be properly assessed in this review, because only two studies used neutral primes. So, although the evidence indicates that semantic priming is automatic, further studies are needed in order to determine whether it cannot also be strategic.

\section{Does Type of Semantic Relationship Matter?}

Collins and Quillian (1969) made hierarchical categorical relationships the basis for organization of knowledge in one of the earliest semantic network models. Perhaps for this reason, studies of semantic priming have tended to focus on category relationships. However, there is evidence that semantic knowledge is organized on the basis of other forms of semantic relationships as well. For example, different kinds of semantic relationships may be impaired in aphasias and Alzheimer's disease (Chertkow, Bub, \& Seidenberg, 1989; Moss, Tyler, Hodges, \& Patterson, 1995; Moss, Tyler, \& Jennings, 1997; Tyler \& Moss, 1997). Moss, Tyler, et al. (1995) and Tyler and Moss (1997) found that functional properties are relatively impervious to breakdown in semantic dementia and aphasia, unlike other types of semantic relationships, including categorical ones. This might mean that functional information is more central to meaning than other types of relationships. Also, some network models of semantic knowledge have similarity or overlapping features rather than categories as the major structural organizing principle (Masson, 1995; McRae \& Boisvert, 1998; McRae, de Sa, \& Seidenberg, 1997). According to these models, types of semantic relationships other than categorical ones can be salient. If these other semantic relationships are encoded in the lexicon, their comparative centrality to word meaning might be evident in relative differences in the effect sizes of different types of semantic relationships.

Unfortunately, many of the studies in the review did not control for type of semantic relationship, and of those that did, a majority used category coordinates as primetarget pairs (e.g., bronze-gold). For other types of relationships, there were sometimes so few observations (and sometimes just from one laboratory) that it would be very premature to draw any conclusions about differences in type of relationships. More research is needed on the priming effects of relationships other than coordinates. But as an indication of where potentially interesting differences might lie, the available effect sizes for different types of semantic relationships are reported here.

A variety of semantic relationships were investigated, including synonyms, antonyms, script relations (e.g., theater-play) and perceptually related primes and targets. Perceptually related pairs either referred to objects of similar shape, as in snake-river (Pecher, Zeelenberg, \& Raaijmakers, 1998), or to object-attribute pairs in which the attribute was a perceptually salient feature of the object, as in snake-long (Lucas, in press). What various researchers called functionally related items were generally instrument/action pairs (e.g., broom-sweep). Although Moss, Ostrin, Tyler, and Marslen-Wilson (1995) included script relationships in the category of functional relationships, other researchers did not. Since Moss, Ostrin, et al. reported means for script and instrument relationships separately, they are kept separate in the following analysis. Finally, some researchers (Keil, 1989; Murphy \& Medin, 1985) have argued that conceptual knowledge is organized around theories that resemble scientific theories, and that word meanings based on such theories incorporate properties that are essential to the constitution of the natural kind object to which the word refers. On this view, for example, CALCIUM is an essential property of MILK since a substance cannot be milk without containing calcium, except under very unusual circumstances. Prime-target pairs related by essential properties constitute a final category of semantic relationship.

Coordinates had an average $d$ of .23 (57 observations) and .22 (16 studies). Synonyms, antonyms, and script relations all had effect sizes in a similar range. For synonyms, the average $d$ was .21 ( 7 observations) and .20 ( 2 studies); for antonyms, the average $d$ was .20 by both observations (5) and studies (1); for script relations, it was .27 by both observations (3) and studies (1). Functional relationships, which included instrument relationships, gave rise to much larger effects (average $d=.55,5 \mathrm{ob}$ servations, and $.46,3$ studies), supporting Tyler and Moss's (1997) proposal that functional relationships are central to meaning. Perceptually related prime-target 
pairs, however, were unusually low in effect size (average $d=.05$ by both observations [10] and studies [2]), as were pairs related by essential features (average $d=.07$ by both observations [4] and studies [1]). These low effect sizes are surprising, given the supposed importance of the latter types of features to word meaning. Again, more work will be required in order to replicate these differences, since the unusually low and unusually high effect sizes were the result of very few observations.

\section{Is There an Associative "Boost"?}

Moss, Ostrin, et al. (1995) have suggested that priming might be greater for word pairs that are both associatively and semantically related than for word pairs that are only semantically related, an effect they call the associative boost. The inclusion of both associatively and semantically related primes in some studies made it possible to determine whether there is an associative boost. To get a $d$ value for the booster effect, the $d$ for the targets that were only semantically related was subtracted from the $d$ for targets that were associatively and semantically related. This measure was calculated only for experiments in which the semantic types of the associative and semantic pairs were equated so that the addition of an associative relation to the semantic relation made for a meaningful comparison. A total of 46 observations across nine studies fit these criteria.

Adding association to a semantic relationship increased the size of the priming effect .24 by observations and .26 by studies, on the average. In other words, the associative boost just about doubled the effect size over that found with purely semantic relationships. So there is some evidence that the addition of an associative bond does have a positive effect on lexical access over and above that provided by a semantic relationship alone.

\section{Do Representational Differences Give Rise to Differences in Associative and Semantic Priming?}

The results of the meta-analysis show that purely semantic relationships between words can be responsible for automatic priming, indicating that these relationships are encoded in the lexicon and are automatically accessed whenever a word is recognized. The evidence for an associative boost further suggests that the association bond adds something over and above the purely semantic. Earlier, one possible source of differences between semantic and associative priming, attentional versus automatic processing, was considered and tentatively dismissed for lack of strong evidence. But even if there are no differences in the types of attentional processing undergone by the two types of relationships, differences in representation may be able to explain the source of at least some differences between the two relationships, notably the source of the associative boost. Evaluation of this possibility requires some discussion about what the terms associative and semantic refer to.

Semantic is generally understood to refer to any kind of meaning relation. Vague as this definition is, unfortu- nately, it does capture the imprecision in how semantics has been construed in this literature. In selection of stimuli, the preferred meaning relations have typically been categorical, as noted earlier; recently, investigations of semantic relations have included more varied relationships such as functional, script-based, and perceptual relations, among others. Associative relationships, on the other hand, are usually defined by the measure used to establish the relationship-the word association test that measures the ease with which one word comes to mind given another.

But what gives rise to word associations? On one view, associative relationships are often simply stronger semantic relationships; the associative response is elicited because of the very strong semantic relationship between words (McRae \& Boisvert, 1998). In support of this hypothesis, Thompson-Schill et al. (1998) presented evidence that associatively related prime-target pairs in and of themselves do not automatically prime unless they are also semantically related. They argued that earlier failures to find automatic priming for semantically related words were due to low semantic similarity among pairs. On this view, the associative "boost" would arise simply from the stronger semantic relationship that typically holds among associated word pairs.

There are good reasons for entertaining this hypothesis. After all, we have evolved to notice, remember, and exploit meaningful relationships in our environment; therefore, it is likely that these meaningful relationships are the primary glue holding together our semantic networks. And most associative relationships are also semantic. Because there has been a tendency to take "semantic" relationships to refer to categorical relationships only, the semantic relationships among associates have sometimes been missed by researchers attempting to study purely associative priming. For example, needle-thread might be deemed a purely associative relationship because there is no categorical relationship between the two words; however, there is a meaningful instrumental relationship between them (Moss, Ostrin, et al., 1995).

The association as semantic relationship hypothesis is hard to assess at this time, for a variety of reasons. Although all authors of the studies included in this metaanalysis assiduously collected association norms on stimuli, fewer collected norms on the strength of semantic relationship. Even if most authors had collected such norms, it would be difficult to equate strength of semantic relationship across studies. Unlike association norms, for which the task is fairly standard, authors had different ways of posing the question about semantic relationship; they used different measurement scales, and so forth. Resolution of this issue requires not just more attention to this variable on the part of researchers but also the development and acceptance of standard similarity norms that can be compared across studies in the same way association norms now are.

An alternative hypothesis about the source of the associative boost involves a distinctive role for associative 
connections over and above their semantic role. In an influential renaissance of Hume's proposal that associative relationships can originate in contiguity - that is, in the frequent co-occurrence of ideas in space and timeFodor (1983) proposed that word associations are due to the frequent co-occurrence of words in language use. On this view, the co-occurrence frequency of words in discourse is reflected in the connections between the corresponding lexical representations in the mental lexicon. Earlier, we saw that this proposal led to the intralexical priming hypothesis, in which co-occurrence bonds give rise to priming, but semantic relationships are encoded in a separate conceptual network, which cannot provide feedback to the associative lexical network. This hypothesis has not been supported by this meta-analysis, which provides evidence for a more integrated lexicon. But, if priming can be the result of purely semantic relationships, it might not be exclusively so. That is, priming might be a function of connections formed by the cooccurrence of words in discourse as well as a function of semantic relationships. If so, a hybrid model could explain the associative "boost." Moss, Ostrin, et al. (1995) hypothesized that much of priming is semantically based but can receive a boost from the additional priming provided by a co-occurrence relationship.

Finding evidence for this hypothesis requires finding an effect that is the converse of semantic priming without association: purely co-occurrence priming without a semantic relationship. The latter effect is difficult to uncover, because, as has just been noted, associations are often also semantic. Also, efforts to determine whether pure co-occurrence relationships can prime have been few and the results equivocal. McKoon and Ratcliff $(1979,1986)$ found priming for episodically studied prime-target pairs that were unrelated semantically. McKoon and Ratcliff (1992) developed a measure of cooccurrence called the "t value," which measures the degree of co-occurrence in a six-word window. Pairs with high $t$ values co-occur in that window more frequently than pairs with lower $t$ values. The high $t$ value pairs in Experiment 3 of their study were not associated; nonetheless, they showed priming. However, semantic relationships could have been responsible for the priming that they found. Quite a few of their stimuli seem to have had a noncategorical semantic relationship (e.g., young-kids; kitchen-knife).

Other attempts to find evidence for co-occurrence priming have not been so successful. Neely and Durgunoğlu (1985) and Carroll and Kirsner (1982) reported failures to find priming for episodic co-occurrence relations. Durgunoğlu and Neely (1987), attempting to replicate McKoon and Ratcliff's successes, found episodic priming only when all studied items were words and when no semantic extralist primes were used. They argue that strategic processing may have been at work in the earlier studies.

Dagenbach, Horst, and Carr (1990) suggested that a problem with any attempt to study episodic priming is that no experiment can hope to mimic the number of episodes in real life in which associated pairs are encountered. Any failure to find episodic priming, then, could be attributed to lack of a sufficient number of training episodes. The few studies which investigated episodic priming usually presented a to-be-remembered pair just once for some extended study period. Dagenbach et al. were unable to find priming under these conditions, but when subjects were given 5 weeks worth of training on the pairs, they did find priming. However, this extensive training worked only for meaningful pairs. This last effect is intriguing, indicating that associations develop only among meaningful word pairs. But there are reasons to question this result. It is, after all, a null effect, and the sample size was very small $(N=12)$. However, more support for Dagenbach's conclusions can be found in a study that took a different approach to the problem. Instead of looking for episodic priming, de Mornay Davies (1998) studied pure co-occurrence relationships that are encountered frequently in natural language use, such as those found in compound words (e.g., honeymoon). He found no priming for targets in the pairs formed from such compounds.

The failure to find co-occurrence effects is interesting in light of two recent models of semantic knowledge, LSA, or latent semantic analysis (Landauer, 1999; Landauer \& Dumais, 1997), and HAL, or hyperspace analogue to language (Burgess, 1998; Lund \& Burgess, 1996). The research just reviewed defined co-occurrence locally; that is, word pairs were considered to co-occur if the experimenters directly linked them or if they cooccurred often in strings of text. LSA and HAL, however, have attained striking success in simulating priming by using global measures of co-occurrence. Starting with local contextual contiguity among words in a given corpus, the models build a vector representation of each word in the corpus. The vectors constitute points in a very high-dimensional semantic space. Through constraint satisfaction, this space can capture indirect associations among words that do not explicitly co-occur in texts (Landauer \& Dumais, 1997) and that embody generalizations formed from the entire contextual history of the word along with all the other words encountered by the model (Burgess, 1998). The resulting semantic space embodies abstract and generalized similarities among words. As a consequence words that may seldom if ever co-occur locally (e.g., road and street) may nevertheless have very similar vector representations. Although in both models (global) co-occurrence plays a seminal role in the formation of semantic relationships, both models hypothesize that semantic relationships (defined by the high-dimensional semantic space) are the primary source of priming phenomena, not the co-occurrence relationships per se. Indeed, in a simulation of the semantic priming effect, Lund, Burgess, and Atchley (1995) failed to find priming for word pairs that were associatively but not semantically related. Impressive as these simulations are, whether or not HAL and LSA constitute 
viable and complete psychological models of semantic knowledge awaits further study (see Barsalou, 1999, for a discussion of the limitations of these models). Nonetheless this work casts further doubt on the possibility of cooccurrence connections (at least local co-occurrence connections) as a primary source of associative priming.

\section{CONCLUSIONS}

The results of the meta-analysis indicate that semantic priming can occur automatically and without association and for varied types of semantic relationships. Moreover, strongly associated word pairs can lead to a boost in priming over and above the effect of semantic relationship alone. Whether this associative "boost" is due to a particularly strong semantic relationship among associated words or to the addition of a co-occurrence relationship remains unclear. Similarly, it is unclear whether semantic and associative priming differ in attentional processing, with semantic priming being exclusively automatic and associative priming being exclusively strategic. The results of the meta-analysis do, however, strongly support the interaction of lexical and semantic information over a modular view.

The results also carry implications for popular models of lexical access that make predictions about semantic and associative priming: the spreading activation model, the compound cue model, and various distributed models.

Distributed models of semantic representation, based as they are on semantic similarity, are clearly supported by the data. In a distributed model of semantic representation, word meaning is represented by a pattern of activation over a large number of units representing features or microfeatures. Related words share many of the same features. On this view, a prime facilitates the processing of the target by activating many of the same features that the target will. These models naturally predict semantic priming without association: the more the overlap between the patterns of activation for two words, the more likely that priming will occur and the stronger the effect. Should it turn out that further investigation finds that purely associative (i.e., co-occurrence) priming also contributes to the priming effect, distributed networks have been shown to be flexible enough to accommodate cooccurrence as well as semantic relationships (Moss, Hare, Day, \& Tyler, 1994; Plaut, 1995).

Any model that predicts that associative relationships are the primary source of priming phenomena does not fare as well. The evidence indicates that purely semantic relationships are the best predictors of priming, particularly automatic priming. This would seem to pose insoluble difficulties for spreading activation models, in which activation is a function of associative relationships-even though such models make the right predictions about the interactive nature of lexical and conceptual networks. It is worth pointing out, however, that in the most frequently cited network model of this sort (Collins \& Loftus, 1975), links among items in the spreading activation network were supposed to be a function of semantic similarity based on shared properties, not on association. Nonetheless, several papers in this review cited the Collins and Loftus model as an example of an associative network. Others reported that semantic similarity was one basis for connectivity in that network but that associative relationships also played an important role. Perhaps this misunderstanding arose because Collins and Loftus themselves sometimes referred to the "associated" concepts in their network, at a time when distinctions between semantic and associative relationships were typically not made. Careful reading of their paper, however, indicates that "associated' pairs were viewed as associated on the basis of the closeness of semantic relationship, and not contiguity or free association production frequency. In the emphasis on semantic similarity as an organizing principle, the Collins and Loftus spreading activation model resembles distributed models, even though there are many important differences between modern distributed networks and the Collins and Loftus network (e.g., weighted connections, constraint satisfaction, the role of microfeatures, etc.). Nonetheless, it remains true that many researchers construe spreading activation networks as primarily associative networks and that several models of spreading activation networks are primarily associative (e.g., Anderson, 1983; McNamara, 1992). On that basis, spreading activation models make poor models of priming.

The compound cue model (McKoon \& Ratcliff, 1989, 1992; Ratcliff \& McKoon, 1995) differs from the other two models in that there is no activation. According to the compound cue model, the prime and target form a compound cue in short-term memory that is used to search long-term memory. The greater the familiarity of the compound, the easier the access to the target in longterm memory. Familiarity is a function of "the frequency with which the subjects in our experiments have encountered or processed the compound in past experience" (McKoon \& Ratcliff, 1992, p. 1163). This approach seems most compatible with a co-occurrence account of priming, and, in fact, MeKoon and Ratcliff (1992) used local co-occurrence as the operational measure of association value in their test of semantic priming. But, as noted earlier, the evidence for local co-occurrence as a source of priming is weak. McKoon and Ratcliff (1992) claim that explanations of priming based on semantic relatedness alone can also be accommodated in their model, but the model has, so far, not been focused on that aspect of word relationships. In comparison with distributed models, which have had semantic similarity as the basis for priming from the start, and which have a fairly sophisticated account of how semantic relationships are encoded in the lexicon, the compound cue model does not provide as complete or satisfying an account of semantic priming without association.

Finally, the meta-analysis reveals many areas in which further research is needed. One moderator variable I would have liked to analyze was strength of semantic re- 
lationship. Unfortunately, this information was only infrequently gathered, and there was no way to compare estimates of strength of semantic relationship across studies (as discussed on p. 625 above). There is evidence that this variable could very well make a significant difference in effect size across studies from work reported in McRae et al. (1997). They asked subjects to rate similarity of their own prime-target pairs as well as those from Shelton and Martin (1992), using the same 1-9 measurement scale for both. The average ratings for the Shelton and Martin stimuli were almost half as large as those for McRae et al.'s. Next they ran the two sets of stimuli in a CLD paradigm and found priming for the more similar stimuli $(d=.55)$. The less similar stimuli did not show priming by significance testing, although there was a small semantic priming effect $(d=.14)$. More studies need to directly manipulate strength of semantic relationship as McRae et al. did to determine whether the strength variable might be responsible for some of the failures to find significant priming effects. It is also possible that the lower average effect size for semantically related pairs in comparison with associatively related pairs is partially, if not entirely, explained by this variable, as proponents of the association-as-semanticrelationship hypothesis, discussed in the previous section, would argue.

Another area in need of greater attention concerns the methods for determining that semantically related pairs are not also associated. As noted in the Method section, there was no consensus on the best way to do this. This problem is exacerbated by the uncertainty over what norms of association measure one should use: strong semantic relationships, or co-occurrence. As discussed on pp. 625-627 above, there is currently no strong evidence for either hypothesis. Finding consensus will be further complicated by a particularly difficult problem: unlike in attempts to determine the degree of semantic or associative relationship among words, which yield a positive value, the search for a nonassociative relationship is a search for a null effect. But failure to find an association does not mean that it is not there; the technique could be flawed or of poor resolution (McKoon \& Ratcliff, 1992). Until these issues are resolved, researchers should make explicit their justification for using specific norms or techniques for gathering norms.

We also need further investigations into the finegrained structure of the lexicon. Which types of semantic relationships are more likely to prime, and what can these differences tell us about the relative importance of different property types to word meaning? To date, category coordinates have been the primary focus of research, with too little attention paid to other types of semantic relationships such as the perceptual, essential, and functional relations. Even less attention has been addressed to priming of lexical relationships that have a syntactic component, such as priming among words that instantiate thematic roles or priming among function words and the content words with which they are typically associated.

One promising area of research not directly addressed in this review concerns the differing roles of the hemispheres in lexical processing. Chiarello and her colleagues have presented evidence that suggests that although semantic information is similarly represented in the two hemispheres, spreading activation in the right hemisphere is more diffuse than the more focused and limited activation of the left hemisphere (Chiarello, Burgess, Richards, \& Pollack, 1990; Chiarello \& Richards, 1992). They found that related but nonassociated words were more readily primed in the right hemisphere than the left. Unfortunately, this hypothesis has seldom been investigated. Researchers controlled for associative relationships in only two of the studies from which effect sizes could be calculated for this meta-analysis (Abernethy \& Coney, 1996; Chiarello \& Richards, 1992), and the findings contradict each other. This may be, as Abernethy and Coney assert, because of differences in the choice of baseline between the two studies, but there are simply not enough observations in this area to be certain about that. More work on hemispheric differences in semantic priming is needed to resolve this issue.

Finally, the lessons of the priming literature for isolated words need to be integrated with results from the sentence processing literature. The lexicon exists, after all, to serve comprehension and production of discourse. Unfortunately, researchers typically do not control for associative versus semantic relationship of prime and target in sentence processing studies, so that more attention to the type and strength of relationship is needed in those studies (Lucas, 1999). But sentence processing is considerably more complex than single word priming. Priming builds across several words in a sentence and involves constraints at levels beyond the lexical; it is not clear how all these constraints affect automatic priming of the target. For example, one proposal is that automatic priming may be affected by the meaningfulness of the discourse. Hess, Foss, and Carroll (1995) argue that experiments done with isolated words are useful but of limited utility in helping us figure out how the lexicon works in discourse comprehension, because humans are always seeking coherence and meaning. They found that when a priming task was embedded in a discourse context, global contexts (established by the topic or focus of the scenario described by the discourse) rather than local contexts (predictable agent-action relationships) determined whether there would be priming. Hess et al. concluded that subjects seek meaning at the highest level possible; when the only information available consists of isolated words, they seek whatever coherence relationships they can within the word pairs. These conclusions suggest that the study of isolated word pairs can provide us with a baseline of lexical organization against which to chart the dynamic flux of semantic relationships in language use. But we need to go beyond the word pair to 
consider the task and the context if we are to build a complete and ecologically valid view of the lexicon and its operations.

\section{REFERENCES}

(An asterisk $\left[{ }^{*}\right]$ before an article indicates one of the priming studies included in the meta-analysis.)

*Abernethy, M., \& Coney, J. (1996). Semantic category priming in the left cerebral hemisphere. Neuropsychologia, 34, 339-350.

ANDERSON, J. R. (1983). The architecture of cognition. Cambridge, MA: Harvard University Press.

Balota, D. A. (1983). Automatic semantic activation and episodic memory encoding. Journal of Verbal Learning \& Verbal Behavior, 22, 88-104.

Balota, D. A., \& Chumbley, J. I. (1984). Are lexical decisions a good measure of lexical access? The role of word frequency in the neglected decision stage. Journal of Experimental Psychology: Human Perception \& Performance, 10, 340-357.

Barsalou, L. W. (1999). Perceptual symbol systems. Behavioral \& Brain Sciences, 22, 577-660.

BORONAT, C. B. (1998). The relationship of attention and semantic priming: Semantic priming is conditionally automatic. Unpublished doctoral dissertation, University of Illinois, Urbana-Champaign.

BURGESS, C. (1998). From simple associations to the building blocks of language: Modeling meaning in memory with the HAL model. Behavior Research Methods, Instruments, \& Computers, 30, 188198.

Carroll, M., \& KirSner, K. (1982). Context and repetition effects in lexical decision and recognition memory. Journal of Verbal Learning \& Verbal Behavior, 21, 55-69.

*Chertkow, H., Bub, D., \& Seidenberg, M. (1989). Priming and semantic memory loss in Alzheimer's disease. Brain \& Language, 36, 420-466.

Chiarello, C., Burgess, C., Richards, L., \& Pollock, A. (1990). Semantic and associative priming in the cerebral hemispheres: Some words do, some words don't ... Sometimes, some places. Brain \& Language, 38, 75-104

*Chiarello, C.. \& Richards. L. (1992). Another look at categorical priming in the cerebral hemispheres. Neuropsvchologia, 30, 381 . 392.

COHEN, J. (1977). Statistical power analysis for the behavioral sciences. New York: Academic Press.

Collins, A. M., \& Loftus, E. F. (1975). A spreading-activation theory of semantic processing. Psychological Review, 82, 407-428

Collins, A. M., \& Quillian, M. R. (1969). Retrieval time from semantic memory. Journal of Verbal Learning \& Verbal Behavior, $\mathbf{8}$, 240-248.

Dagenbach, D., Horst, S., \& Cark, T. H. (1990). Adding new information to semantic memory: How much learning is enough to produce automatic priming? Journal of Experimental Psychology: Learning, Memory, \& Cognition, 16, 581-591.

DeGroot, A. M. B. (1984). Primed lexical decision: Combined effects of the proportion of related prime-target pairs and the stimulus-onset asynchrony of prime and target. Quarterly Journal of Experimental Psychology, 36A, 253-280.

DE Mornay Davies, P. (1998). Automatic semantic priming: The contribution of lexical- and semantic-level processes. European Journal of Cognitive Psychology, 10, 389-412.

*DJosominaRDjo, M. (1997). Association: How does it differ from semantic relatedness? Unpublished doctoral dissertation, Ohio University.

DurgunoǦlu, A. Y., \& Neely, J. H. (1987). On obtaining episodic priming in a lexical decision task following paired-associate learning. Journal of Experimental Psychology, 13, 206-222.

*FisCHLER, I. (1977). Semantic facilitation without association in a lexical decision task. Memory \& Cognition, 5, 335-339.

FODOR, J. A. (1983). The modularity of mind: An essay on faculty psychology. Cambridge, MA: MIT Press.
FORSTER, K. I. (1979). Levels of processing and the structure of the language processor. In W. E. Cooper \& E. C. T. Walker (Eds.), Sentence processing: Psycholinguistic studies presented to Merrill Garrett (pp. 257-287). Cambridge, MA: MIT Press.

Glass, G. V., McGaw, B., \& SMITH, M. L. (1981). Meta-analysis in social research. Beverly Hills, CA: Sage.

HEDGES, L. V., \& OLKIN, I. (1985). Statistical methods for metaanalysis. New York: Academic Press.

Henik, A., Friedrich, F. J., \& Kellogg, W. A. (1983). The dependence of semantic relatedness effects upon prime processing. Memory \& Cognition, 11, 366-373.

Hess, D. J., Foss, D. J., \& Carroll, P. (1995). Effects of global and local context on lexical processing during language comprehension. Journal of Experimental Psychology: General, 124. 62-82.

HodGSON, J. M. (1991). Informational constraints on pre-lexical priming. Language \& Cognitive Processes, 6, 169-205.

HUTTENLOCHER, J., \& KUBICEK, L. (1983). The source of relatedness effects on naming latency. Journal of Experimental Psychology: Learning, Memory, \& Cognition, 9, 486-496.

*KaCINIK, N.A. (1998). The prime task effect: An investigation of semantic vs. associative priming and the activation blocking account. Unpublished master's thesis, University of Western Ontario.

KeEFE, D. E., \& NeEly, J. H. (1990). Semantic priming in the pronunciation task: The role of prospective prime-generated expectancies. Memory \& Cognition, 18, 289-298.

KEIL, F. C. (1989). Concepts, kinds, and conceptual development. Cambridge, MA: MIT Press.

*KrUESI, E. E. (1979). The role of associations and semantic similarity in facilitating the lexical decision. Unpublished doctoral dissertation. University of California, Berkeley.

LANDAUER, T. K. (1999). Latent semantic analysis (LSA), a disembodied learning machine, acquires human word meaning vicariously from language alone. Behavioral \& Brain Sciences, 22, 624625.

Landauer, T. K., \& Dumais, S. T. (1997). A solution to Plato's problem: The latent semantic analysis theory of acquisition, induction, and representation of knowledge. Psychological Bulletin, 104, 211240.

Light, R. J., \& Pillemer, D. B. (1984). Summing up: The science of reviewing research. Cambridge, MA: Harvard University Press.

LoGAN, G. D. (1988). Toward an instance theory of automatization. Psychological Review, 95, 492-527.

LuCAS, M. (1999). Context effects in lexical access: A meta-analysis. Memory \& Cognition, 27, 385-398.

*LuCAS, M. (in press). Essential and perceptual attributes of words in reflective and on-line processing. Journal of Psycholinguistic Research.

Lund, K., \& Burgess, C. (1996). Producing high-dimensional semantic spaces from lexical co-occurrence. Behavior Research Methods, Instruments, \& Computers, 28, 203-208.

Lund, K., Burgess, C., \& Atchley, R. A. (1995). Semantic and associative priming in high-dimensional semantic space. In Proceedings of the Cognitive Science Society (pp. 660-665). Hillsdale, NJ: Erlbaum.

Masson, M. E. J. (1995). A distributed memory model of semantic priming. Journal of Experimental Psychology: Learning, Memory, \& Cognition, 21, 3-23.

McKoon, G., \& Ratcliff, R. (1979). Priming in episodic and semantic memory. Journal of Verbal Learning \& Verbal Behavior, 18, 463-480.

MCKoON, G., \& RaTCLIFF, R. (1986). Automatic activation of episodic information in a semantic memory task. Journal of Experimental Psychology: Learning, Memory, \& Cognition, 12, 108-115.

McKoon, G., \& Ratcliff, R. (1989). Assessing the occurrence of elaborative inference with recognition: Compatibility checking vs. compound cue theory. Journal of Memory \& Language $\% 28,547$ 563.

MCKOON, G., \& RATCLIFF, R. (1992). Spreading activation versus compound cue accounts of priming: Mediated priming revisited. Journal of Experimental Psychology: Learning, Memory, \& Cognition, 18, $1155-1172$ 
MCNamara, T. P. (1992). Priming and constraints it places on theories of memory and retrieval. Psychological Review, 99, 650-662.

MCNAmara, T. P., \& Altarriba, J. (1988). Depth of spreading activation revisited: Semantic mediated priming occurs in lexical decisions. Journal of Memory \& Language, 27, 545-559.

*MCRAE, K., \& BoisverT, S. (1998). Automatic semantic similarity priming. Journal of Experimental Psychology: Learning, Memory, \& Cognition, 24, 558-572.

MCRaE, K., DE SA, V. R., \& Seidenberg, M. S. (1997). On the nature and scope of featural representations of word meaning. Journal of Experimental Psychology: General, 126, 99-130.

Meyer, D. E., \& Schvaneveldt, R. W. (1971). Facilitation in recognizing pairs of words: Evidence of a dependence between retrieval operations. Journal of Experimental Psychology, 90, 227-234.

Meyer, D. E., Schvaneveldt, R. W., \& Ruddy, M. G. (1975). Loci of contextual effects in visual word recognition. In P. M. A. Rabbit \& S. Dornic (Eds.), Attention and performance $V$ (pp. 98-118). New York: Academic Press.

Moss, H. E., Hare, M. L., Day, P., \& Tyler, L. K. (1994). A distributed memory model of the associative boost in priming. Connection Science, 6, 413-427.

Moss, H. E., McCormick, S. F., \& TYLer, L. K. (1997). The time course of activation of semantic information during spoken word recognition. Language \& Cognitive Processes, 12, 698-731.

*Moss, H. E., Ostrin, R. K., Tyler, L. K., \& Marslen-Wilson, W. D. (1995). Accessing different types of lexical semantic information: Evidence from priming. Journal of Experimental Psychology: Learning, Memory, \& Cognition, 21, 863-883.

Moss, H. E., Tyler, L. K., Hodges, J. R., \& Patterson, K. (1995). Exploring the loss of semantic memory in semantic dementia: Evidence from a primed monitoring study. Neuropsychology, 9, 16-26

Moss, H. E., TYleR, L. K., \& JenNings, F. (1997). When leopards lose their spots: Knowledge of visual properties in category-specific deficits for living things. Cognitive Neuropsychology, 14, 901-950.

MurPhy, G. L., \& MEDIN, D. L. (1985). The role of theories in conceptual coherence. Psychological Review, 92, 289-316.

*NATION, K., \& SNOWLING, M. J. (1999). Developmental differences in sensitivity to semantic relations among good and poor comprehenders: Evidence from semantic priming. Cognition, 70, B1-B13.

NEELY, J. H. (1977). Semantic priming and retrieval from lexical memory: Roles of inhibitionless spreading activation and limited-capacity attention. Journal of Experimental Psychology: General, 106, 226254.

NEELY, J. H. (1991). Semantic priming effects in visual word recognition: A selective review of current findings and theories. In D. Besner \& G. W. Humphreys (Eds.), In Basic processes in reading: Visual word recognition (pp. 264-336). Hillsdale, NJ: Erlbaum.

Neely, J. H., \& DurgunoĞlu, A. Y. (1985). Dissociative episodic and semantic priming effects in episodic recognition and lexical decision tasks. Journal of Memory \& Language, 24, 466-489.

*Ostrin, R. K. \& TyLer, L. K. (1993). Automatic access to lexical semantics in aphasia: Evidence from semantic and associative priming. Brain \& Language, 45, 147-159.

PAAP, K. R., \& Newsome, S. L. (1981). Parafoveal information is not sufficient to produce semantic or visual priming. Perception $\&$ Psychophysics, 29, 457-466.

*Pecher, D., Zeelenberg, R., \& RaAijmakers, J. G. W. (1998). Does pizza prime coin? Perceptual priming in lexical decision and pronunciation. Journal of Memory \& Language, 38, 401-418.

*Perea, M., \& Gotor, A. (1997). Associative and semantic priming effects occur at very short stimulus-onset asynchronies in lexical decision and naming. Cognition, 62, 223-240.

*Perea, M., Gotor, A., \& Nacher, M. J. (1997). Efectos de facilitación asociativa vs. semántica con una breve asincronía estimular senal-test. Psicothema, 9, 509-517.

*Perea, M., \& Rosa, E. (1998) The effects of associative and semantic priming in the lexical decision task. Unpublished manuscript.

*Perfetti, C. A. \& TAN, L. H. (1998). Time course of graphic, phonological, and semantic activation in Chinese character identification. Journal of Experimental Psychology: Learning, Memory, \& Cognition, 24, 101-118.

PlaUt, D. C. (1995). Semantic and associative priming in a distributed attractor network. In Proceedings of the Seventeenth Annual Conference of the Cognitive Science Society (pp. 37-42). Hillsdale, NJ: Erlbaum.

Posner, M. I., \& Snyder, C. R. R. (1975). Attention and cognitive control. In R. L. Solso (Ed.), Information processing and cognition: The Lovola symposium (pp. 55-86). Hillsdale, NJ: Erlbaum.

Ratcliff, R., \& McKoon, G. (1995). Sequential effects in lexical decision: Tests of compound-cue retrieval theory. Journal of Experimental Psychology: Learning, Memory, \& Cognition, 21, 13801388.

Rosenthal, R., \& Rubin, D. B. (1982). Comparing effect sizes of independent studies. Psychological Bulletin, 92, 500-504.

*SCHolten, I. (1991). Semantic and associative priming in aphasic subjects. Unpublished master's thesis, University of Wisconsin, Madison.

*Seidenberg, M. S., Waters, G. S., Sanders, M., \& Langer, P. (1984). Pre- and postlexical loci of contextual effects on word recognition. Memory \& Cognition, 12, 315-328.

*Shelton, J. R. (1993). Semantic priming of lexical and conceptual representations: Manipulating depth of processing of the prime. Unpublished doctoral dissertation, Rice University.

*Shelton, J. R., \& MARTIN, R. C. (1992). How semantic is automatic semantic priming? Journal of Experimental Psychology: Learning Memory, \& Cognition, 18, 1191-1210.

Smith, M. C. (1979). Contextual facilitation in a letter search task depends on how the prime is processed. Journal of Experimental Psychology: Human Perception \& Performance, 5, 239-251.

Stolz, J. A., \& Besner, D. (1999). On the myth of automatic semantic activation in reading. Current Directions in Psychological Science, 8, 61-65.

*ThompSON-Schill, S. L., Kurtz, K. J., \& Gabriell, J. D. E. (1998). Effects of semantic and associative relatedness on automatic priming. Journal of Memory \& Language, 38, 440-458.

TYLER, L. K., \& Moss, H. E. (1997). Functional properties of concepts: Studies of normal and brain-damaged patients. Cognitive Neuropsychology, 14, 511-545.

*Williams, J. N. (1994). The relationship between word meanings in the first and second language: Evidence for a common, but restricted, semantic code. European Journal of Cognitive Psychology, 6, 195-220.

*Williams, J. N. (1996). Is automatic priming semantic? European Journal of Cognitive Psychology, 8, 113-161.

*Zwitserlood, P., \& Schriefers, H. (1995). Effects of sensory information and processing time in spoken-word recognition. Language \& Cognitive Processes, 10, 121-136.

(Manuscript received August 18, 1999; revision accepted for publication April 7, 2000. 